\title{
Optimal Control for an Irrigation Problem with Several Fields and Common Reservoir
}

\author{
Sofia O. Lopes ${ }^{13}$ and Fernando A.C.C. Fontes ${ }^{2}$ \\ ${ }^{1}$ CMAT and Departamento de Matemática e Aplicações, Universidade do Minho, \\ Guimarães, Portugal \\ ${ }^{2}$ Systec-ISR, Faculdade de Engenharia, Universidade do Porto, Porto, Portugal
}

\begin{abstract}
In a previous study, the authors developed the planning of the water used in the irrigation systems of a given farmland in order to ensure that the field cultivation is in a good state of preservation. In this paper, we introduce a model to minimize the water flowing into a reservoir that supplies different fields with different types of crops. This model is described as an optimal control problem where the water flow from a tap and the water used in the fields are the controls. The trajectories are described as the humidity in the soil and the amount of water in the reservoir.
\end{abstract}

\section{Introduction}

According to [13], the water demand in Portugal in the three sectors of activity urban, industry and agriculture amounts to $7500 \times 10^{6} \mathrm{~m}^{3} /$ year. The agriculture is responsible for $80 \%$ of the water demand, most of it in the in the spring and summer periods, and it is estimated that $37.5 \%$ is lost due to various inefficiencies. These facts affect strongly the agriculture production. It is, consequently, relevant to discuss the use of water in these conditions, and try to find the best technical solutions, economic and social, to improve the efficiency of water usage.

The irrigation planning systems here developed are based on the minimization of the volume of water used in irrigation (control), knowing that the variation of water in the soil (trajectory) is given by the hydrologic balance. The connections between an irrigation planning system and an optimal control problems are easily established, however this connection still has lot to be explored, see for example [2], [1] and [5].

A model to optimize the water use in the irrigation of a farm field via optimal control (water flow) that takes into account the evapotranspiration, rainfall, losses by infiltration and runoff was developed in [7]. There, the solution was obtained for an "Yearly Planning" problem considering different weather scenarios with the help of the so called "precipitation factor" that is multiplied by the rainfall monthly average. In [6], the authors present the "Initial Planning Problem" for rainfall: this includes an extra term taking into account the rainfall in the previous time period (this rainfall model was statistically proven to be significant) where a comparison between this new model and the solution knowing the rainfall a priori was shown. A characterization of the solution using the 
necessary conditions of optimality was made in [8], and thereby the numerical results were validated. Most of these results and further details are collected in [11].

In this paper, we developed a model that allows to minimize the water used in a reservoir to supply different fields with different types of crops where the water of precipitation can be collected in a given area, having in mind that is crucial to ensure the good state of preservation of the cultivation field. The main contribution with respect to previous works is precisely the fact that the model includes several different fields that share a common reservoir.

This paper is organized as follows. Section 2 presents the problem formulation. Section 3 describes the numerical model and Section 4 reports numerical results in different scenarios. Concuding remarks are drawn in Section 5.

\section{The Problem}

In order to describe our problem, we start by defining the variables used. The controls are: $v$ total water flow coming from the tap and $u_{j}$ water flow introduced in field $j$ via its irrigation system. The states are: $x_{j}$ water in the soil of field $j$ and $y$ total of amount of water stored in reservoir.

We intend to minimize the total of water flow coming from the tap to a reservoir. The objective function is:

$$
\min \int_{0}^{T} v(t) d t
$$

The variation of water in reservoir is given by:

$$
\dot{y}(t)=v(t)-\sum_{j=1}^{P} A_{j} u_{j}(t)+C g(t),
$$

where $A_{j}$ represents the area of each field $j$ and $C g(t)$ represents collected water in a certain area $C$ coming from the precipitation $g$ in the time $t$.

The variation of water in the soil is given by the hydrologic equation, that is, the variation of water in the soil is equal to what enters (water via irrigation systems and precipitation) minus the loss (evapotranspiration of each crop $h_{j}$ and loss by deep percolation $\beta x_{j}(t)$, a percentage of water that is in the soil).

So,

$$
\dot{x}_{j}(t)=u_{j}+g(t)-h_{j}(t)-\beta x_{j}(t), \forall j=1, \ldots, P
$$

We note that each field has only one crop.

In order to ensure that the crop is in good state of conservation, the water in the each field has to be sufficient to satisfy the hydric needs of each crop $\left(x_{\min }\right)$, that is:

$$
x_{j}(t) \geq x_{\min _{j}} .
$$


The physical limitations of: the amount of water that comes from a tap, the amount of water that comes from the irrigation systems and the reservoir are given, respectively, by:

$$
\begin{aligned}
& y(t) \in\left[0, y_{\max }\right] \\
& u_{j}(t) \in\left[0, M_{j}\right] \\
& v(t) \in\left[0, \sum_{j} A_{j} M_{j}\right]
\end{aligned}
$$

where $y_{\max }$ is the maximum quantity of water in the reservoir and $M_{j}$ is the maximum water flow coming from the tap in each field.

We assume that at the initial time the humidity of the soil in each field and the water in the reservoir are given. Also, the water in the reservoir at the initial time and to the final time are imposed to be equal. So, we assume that:

$$
\begin{aligned}
& x_{j}(0)=x_{0_{j}} \\
& y(0)=y(T)=y_{0}
\end{aligned}
$$

In summary, the optimal control formulation for our problem is:

$$
\begin{array}{ll}
\min \int_{0}^{T} v(t) d t & \\
\text { subject to: } & \\
\dot{x}_{j}(t)=-\beta x_{j}(t)+u_{j}+g(t)-h_{j}(t) \text { a.e. } t \in[0, T], \forall j=1, \ldots, P \\
\dot{y}(t)=v(t)-\sum_{j=1}^{P} A_{j} u_{j}(t)+C g(t) \text { a.e. } t \in[0, T], \\
x_{j}(t) \geq x_{\min _{j}} & \forall t \in[0, T], \forall j=1, \ldots, P \\
y(t) \in\left[0, y_{\max }\right] & \forall t \in[0, T] \\
u_{j}(t) \in\left[0, M_{j}\right] & \text { a.e. }, \forall j=1, \ldots, P \\
v(t) \in\left[0, \sum_{j} A_{j} M_{j}\right] & \text { a.e. } \\
x_{j}(0)=x_{0_{j}} & \forall j=1, \ldots, P \\
y(0)=y(T)=y_{0} &
\end{array}
$$

where the constant parameters involved are the following:

$P$ : number of fields;

$A_{j}$ : Area of field $\mathrm{j}$;

$C$ : Area of the collector of precipitation water;

$\beta$ : the percentage of loss in the soil;

$x_{\min _{j}}$ : hydric needs of the culture in each field (per $m^{2}$ );

$y_{\max }:$ the maximum quantity of water in the reservoir;

$M_{j}$ : maximum water flow coming from the tap of time $t$ in each field (per $m^{2}$ );

$x_{0_{j}}$ : the humidity of the soil in each field (per $\mathrm{m}^{2}$ );

$y_{0}$ : the quantity of water in the reservoir at initial time. 
The decisions variables are the states $\left(x_{j}, y\right)$ and the controls $\left(u_{j}, v\right)$ functions for $j=1, \ldots, P$. These and other time-varying functions are described in the following way:

$x_{j}(t)$ : water in the soil of field $j$ at time $t$ (per $m^{2}$ );

$u_{j}(t)$ : water flow introduced in filed $j$ via its irrigation systems at time $t$ (per $\left.m^{2}\right)$

$y(t)$ : total of amount of water stored in reservoir at time $t$ (maximum capacity $\left.y_{\max }\right)$

$v(t)$ : total water flow coming from the tap at time $t$;

$g(t)$ : the precipitation at time $t$ (per $\left.m^{2}\right)$;

$h_{j}(t)$ : the evapotanspiration at time $t$ of each field (culture) (per $m^{2}$ );

The solution to this optimal control problem can be characterized by necessary conditions of optimality in the form of a maximum principle (see e.g. [14, $11,10,4,9,3]$. In this paper, however, we discuss the numerical results.

\section{Numerical Model for the Irrigation Problem}

In this section, we obtain the numerical solution to our problem transcribing it into a sequence of finite dimensional linear programming problems.

For that, we start by defining a finite number of time instants $i=1, \ldots, N+1$

$$
\begin{aligned}
& x_{i}=x\left(t_{i}\right) \\
& u_{i}=u\left(t_{i}\right)
\end{aligned}
$$

where $t_{i}=(i-1) h$ and $h=T / N$.

We use the Euler-type discretization to the differential equations:

$$
\dot{x}=f(t, x, u) \text { is aproximated to } x_{i}=x_{i-1}+h f\left(t_{i-1}, x_{i-1}, u_{i-1}\right) .
$$

To implement this optimization problem, we use fmincon function of MatLab with the algorithm "active set", by default and the parameter "Tolfun" is considered $1 E-6$.

\subsection{Rainfall models}

To estimate rainfallwe use the monthly rainfall data from Instituto Português do Mar e da Atmosfera ( www.ipma.pt), in the Lisbon area. We defined an average (using the 10 years data) rainfall for each month of the year, the rain monthly average is: $10^{-3} \times$

\begin{tabular}{c|c|c|c|c|c|c|c|c|c|c|c}
$\mathrm{J}$ & $\mathrm{F}$ & $\mathrm{M}$ & $\mathrm{A}$ & $\mathrm{M}$ & $\mathrm{J}$ & $\mathrm{J}$ & $\mathrm{A}$ & $\mathrm{S}$ & $\mathrm{O}$ & $\mathrm{N}$ & $\mathrm{D}$ \\
\hline 111.4 & 94.7 & 80.2 & 57.1 & 29.62 & 18.84 & 1.26 & 7.04 & 30.6 & 127 & 121.98 & $119.3\left(\mathrm{~m}^{3} / \mathrm{month}\right)$
\end{tabular}

Therefore $g\left(t_{i}\right)=$ rain monthly average $\left(t_{i}\right)$. 


\subsection{Evapotranspiration model}

We used the Pennman - Monteith methodology [15] to calculate evapotranspiration of our culture along the year. In order to do so, we use the formulation:

$$
E T\left(t_{i}\right)=K_{c} E T_{0}\left(t_{i}\right),
$$

where $K_{c}=0.825$ is the culture coefficient for the evapotranspiration (in our case potatoes) and $E T_{0}$ is the tabulated reference value of evapotranspiration that we consulted in [12] for the Lisbon region. The evapotranspiration of our culture in Lisbon is given by the following table: $10^{-3} \times$

$\mathrm{J}$
\begin{tabular}{c|c|c|c|c|c|c|c|c|c|c|c} 
& $\mathrm{F}$ & $\mathrm{M}$ & $\mathrm{A}$ & $\mathrm{M}$ & $\mathrm{J}$ & $\mathrm{J}$ & $\mathrm{A}$ & $\mathrm{S}$ & $\mathrm{O}$ & $\mathrm{N}$ & $\mathrm{D}$ \\
\hline 19.8 & 28.0 & 55.27 & 89.1 & 116.32 & 137.77 & 155.92 & 136.95 & 84.97 & 53.62 & 22.27 & $16.5\left(\mathrm{~m}^{3} /\right.$ month $)$
\end{tabular}
So, $h\left(t_{i}\right)=E t\left(t_{i}\right)$.

\section{Results}

To simulate our problem, we assume that:

$$
\begin{array}{lll}
P=3 & T=12 & M_{j}=10 \mathrm{~m}^{3} / \text { month for each field } j \\
y_{\max }=0.05 \times A_{t} & y_{0}=0.01 \times A_{t} & \beta=15 \%
\end{array}
$$

where $A_{t}$ is the total area.

Our first simulation corresponds to a closed reservoir that supplies three fields with the same area in which each field has a different culture. In this example, we consider the three crops: wheat, sugar cane and olive, and the following parameters:

$$
\begin{array}{ll}
x_{\min }=\left[\begin{array}{lll}
0.033 & 0.021 & 0.032
\end{array}\right] & K_{c}=\left[\begin{array}{lll}
0.825 & 0.95 & 0.5
\end{array}\right] \\
x_{0}=5 x_{\min } & C=0
\end{array}
$$

The humidity of the soil (estimated trajectory) and the amount of water used in each field (estimated control) is given in Fig. 1.

As expected, the crops need water between May and September, the months when the water consumption is higher are June, July, the crop that needs less water is olive, and the crop that needs more water is the sugar cane.

The estimated amount of water to irrigate the three field per $m^{2}$ is $0.8330 \mathrm{~m}^{3} /$ year, where in each field we spend the following amount of water per $m^{2}$ :

$u_{\text {wheat }}:=\quad\left[\begin{array}{llllllllllll}0 & 0 & 0 & 0 & 0 & 0.0331 & 0.1323 & 0.1109 & 0.0445 & 0 & 0 & 0\end{array}\right]$;

$u_{\text {sugar cane }}:=\left[\begin{array}{llllllllllll}0 & 0 & 0 & 0 & 0 & 0.0885 & 0.1500 & 0.1262 & 0.0533 & 0 & 0 & 0\end{array}\right] ;$ 


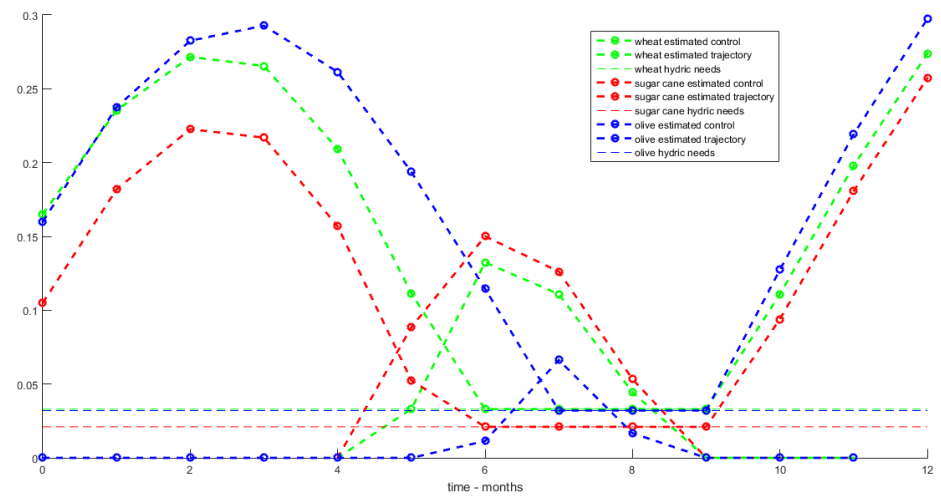

Fig. 1. Humidity in the soil

$u_{\text {olive }}:=\quad\left[\begin{array}{llllllllllll}0 & 0 & 0 & 0 & 0 & 0 & 0.0113 & 0.0662 & 0.0167 & 0 & 0 & 0\end{array}\right]$.

In Fig. 2, we describe the variation of water in the reservoir and the amount of water that comes from a tap.

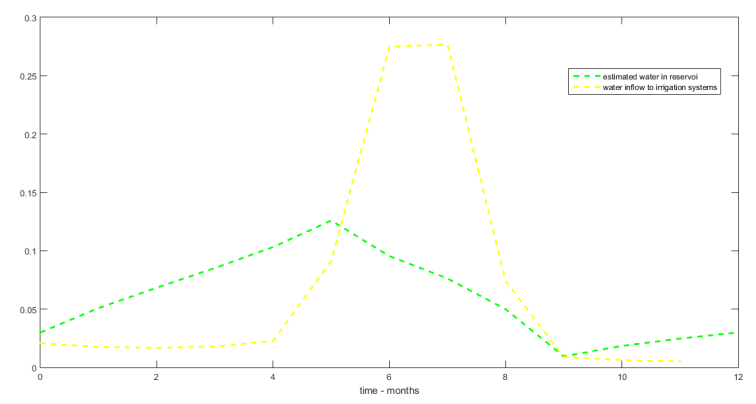

Fig. 2. Water needs

We note that the water in the reservoir at the initial time is equal to the water at the final time. The water that comes from the tap starts to be consumed earlier than the hydric needs of the crops, so the water storage in reservoir increases. June and July are the month when the irrigation takes the highest value, and also when the water in the reservoir decrease. For the water in the reservoir at the initial time to be equal to the one at the final time, the tap is closed later than the needs of the crop, in November. 
In the next simulation, we consider that the fields have different areas. We consider the same initial data of the previous problem and we assume that fields have $1000 \mathrm{~m}^{2}$ of wheat, $750 \mathrm{~m}^{2}$ of sugar cane and $1250 \mathrm{~m}^{2}$ of olive. That is:

$$
\begin{array}{ll}
x_{\min }=\left[\begin{array}{lll}
0.033 & 0.021 & 0.032
\end{array}\right] & K_{c}=\left[\begin{array}{lll}
0.825 & 0.95 & 0.5
\end{array}\right] \quad A=\left[\begin{array}{lll}
1000 & 750 & 1250
\end{array}\right] \\
x_{0}=5 x_{\min } & C=0
\end{array}
$$

The results obtained are reported in Fig. 3:

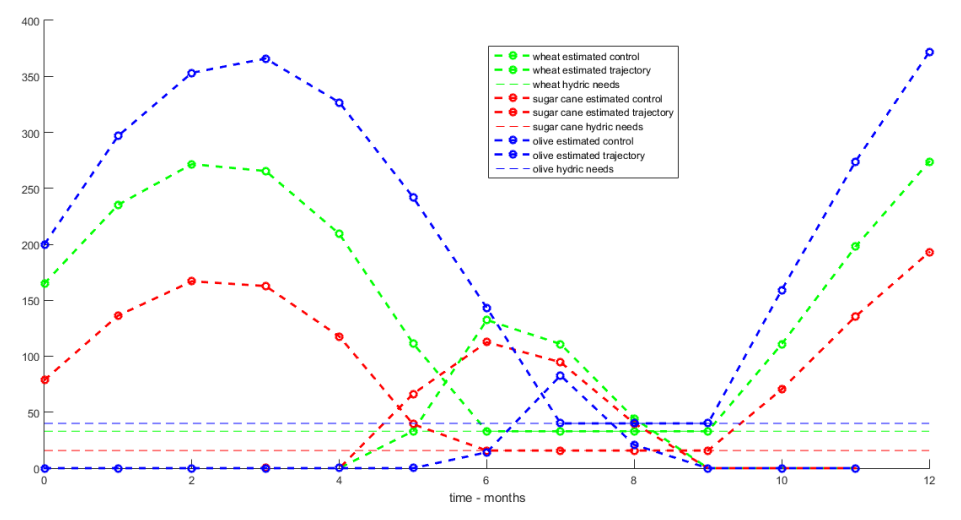

Fig. 3. Humidity in soil with fields having different areas

Comparing these results with the previous ones, we can see that in this case the needs of water for the wheat field are higher than the ones for sugar cane field.

The estimated amount of water to irrigate the three fields is $752.0795 \mathrm{~m}^{3} /$ year, where in each field we spend the following amount of water:

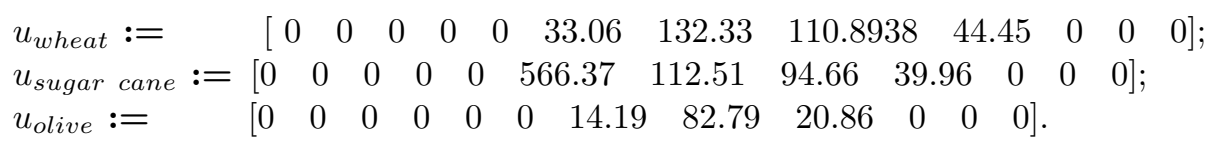

In Fig. 4, we describe the variation of water in the reservoir and the variations on the amount of water that comes from a tap.

We can see that variations of the water in the reservoir and the behaviour of the water that comes from the tap is similar to the previous simulation.

In the next simulation, as in previous ones, we consider the irrigating of three fields with different crops and different areas, however in this case we take 


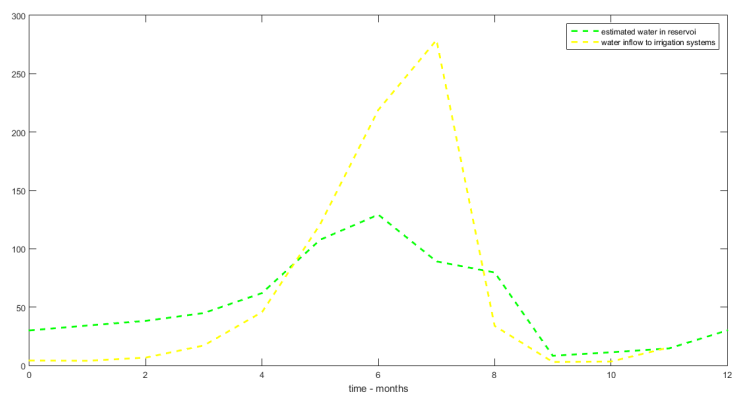

Fig. 4. Water needs where the fields have different areas

advantage of the rainfall information. We assume that reservoir has an area of $30 \mathrm{~m}^{2}$ to collect rain fall. That is,

$$
\begin{array}{lll}
x_{\text {min }}=\left[\begin{array}{lll}
0.033 & 0.021 & 0.032
\end{array}\right] & K_{c}=\left[\begin{array}{lll}
0.825 & 0.95 & 0.5
\end{array}\right] & A=\left[\begin{array}{lll}
1000 & 750 & 1250
\end{array}\right] \\
x_{0}=5 x_{\min } & C=30
\end{array}
$$

The variation of water in the reservoir and the variation of the amount of water that comes from a tap are described in Fig. 5.

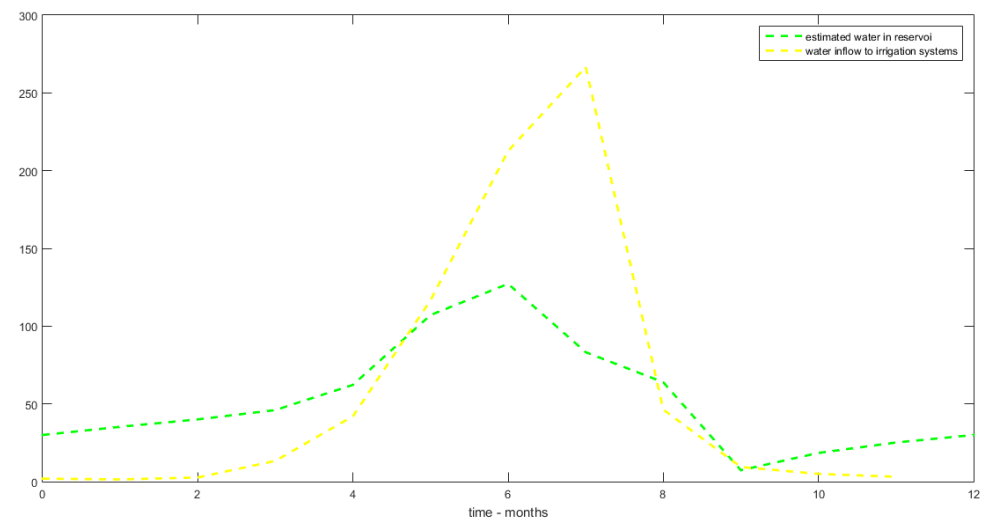

Fig. 5. Water needs with $C=30$

In this case, the amount of water needed is $721.1735 \mathrm{~m}^{3} /$ year, which results in a reduction of $4.1 \%$ in the water needs. We can see that in November the water that comes from the tap is less than the one in the previous simulation. However, the water saving is negligible. We may conclude that a larger collection area of the reservoir would be of advantage. 
In the last simulation, we assume that the top of reservoir has now $200 \mathrm{~m}^{2}$ of area. The variation of water in the reservoir and the variation of the amount of water that comes from a tap is described in Fig. 6.

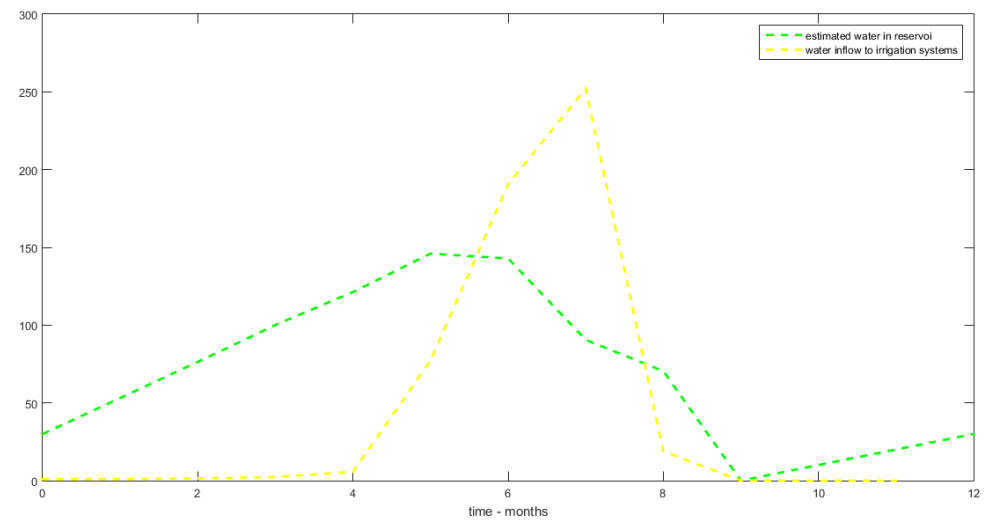

Fig. 6. Water needs with $C=200$

We can see that until April the water from the precipitation is saved in the reservoir and until this month the tap is not opened. The tap is closed earlier than the previous simulations; it is closed in September. In this case the amount of water needed is $550.2994 \mathrm{~m}^{3} /$ year, resulting in a reduction of $26,83 \%$ in the water needs.

\section{Conclusion}

We have developed an irrigation planning model to minimize the water used to supply different fields with different types of crops. The main contribution with respect to previous works is precisely the fact that the model includes several different fields that share a common reservoir, which not only serves as storage, but also is from where the water inflow coming from either tap or rain fall is collected. The optimal control problem formulated is solved numerically for different scenarios of crop area and reservoir capacity.

Acknowledgments. Research carried out while the 2nd author was a visiting scholar at Texas A\&M University, College Station, USA. The support from Texas 
A\&M and FEDER/COMPETE2020-POCI/FCT funds through grants POCI01-0145-FEDER-006933 - SYSTEC, PTDC/EEI-AUT/2933/2014 and

UID/MAT/00013/2013 are acknowledged.

\section{References}

1. Rigon R. Bertoldi, G. GEOTOP A Hydrological Balance Model Technical Descriptionand Programs Guide. Universit Deglli Studi Di Trento, 2004.

2. Cochrane T. A. Krom T. D. Brown, P. D. Optimal on-farm irrigation scheduling with a seasonal water limit using simulated annealing. Agricultural Water Management, 97:892-900, 2010.

3. MdR de Pinho, M. M. Ferreira, and F. A. C. C. Fontes. Unmaximized inclusion necessary conditions for nonconvex constrained optimal control problems. ESAIM: Control, Optimisation and Calculus of Variations, 11(4):614-632, 2005.

4. Fernando ACC Fontes and Sofia O Lopes. Normal forms of necessary conditions for dynamic optimization problems with pathwise inequality constraints. Journal of Mathematical Analysis and applications, 399(1):27-37, 2013.

5. Gandolfi C. Soncini-Sessa R. Agostani D. Galelli, S. Building a metamodel of irrigation district distributed-parameter model. Agricultural Water Management, 97:187-200, 2010.

6. S. Lopes, F. Fontes, R. M. S. Pereira, M. Gonçalves, and G. J. Machado. Irrigation planning: an optimal control approach. International Conference of Numerical Analysis and Applied Mathematics, AIP Conference Proceedings, 1558:622-626, 2013.

7. S. Lopes, F. Fontes, R. M. S. Pereira, and G. J. Machado. Irrigation planning in the context of climate change. Mathematical Models for Engineering Science MMES11, pages 239-244, 2011.

8. Sofia O. Lopes, F. A.C.C. Fontes, Rui M.S. Pereira, MdR de Pinho, and C. Ribeiro. Optimal control for an irrigation planning problem: Characterization of solution and validation of the numerical results. Lecture Notes in Electrical Engineering, 321:157-167, 2015.

9. Sofia O Lopes, FACC Fontes, and MdR de Pinho. On constraint qualifications for nondegenerate necessary conditions of optimality applied to optimal control problems. Discrete and Continuous Dynamical System-A, 29(2), 2011.

10. Sofia O Lopes, Fernando ACC Fontes, and MDR de Pinho. An integral-type constraint qualification to guarantee nondegeneracy of the maximum principle for optimal control problems with state constraints. Systems $\&$ Control Letters, 62(8):686-692, 2013.

11. Sofia O Lopes, Fernando ACC Fontes, Rui MS Pereira, M Rosário de Pinho, and A Manuela Gonçalves. Optimal control applied to an irrigation planning problem. Mathematical Problems in Engineering, 2016.

12. J. R. Raposo. A REGA - dos primitivos regadios as modernas técnicas de rega. Fundação Calouste Gulbenkian, 1996.

13. Pedro Sousa, Luis Ribeiro, and Alda Carvalho. Uso da agua na agricultura 2011. Instituto Nacional de Estatistica, 2011.

14. R. Vinter. Optimal control. Birkhauser, Boston, 2000.

15. I. A. Walter, R. G. Allen, R. Elliott, D. Itenfisu, and et.al. The ASCE standardized reference evapotranspiration equation. Rep. Task Com. on Standardized Reference Evapotranspiration, 2002. 